\title{
A Simulation Study of an Unpolarized Field
}

\author{
Youssef Khmou \\ Sultan Moulay Slimane University, Morocco \\ Khmou.y@gmail.com
}

\begin{abstract}
The electric field of an electromagnetic wave has generally two components perpendicular to the direction of propagation, the relationship between the different characteristics of the field's components give different states of polarization, generally the field can be in polarized state, partially polarized or unpolarized state. In this paper we present a fast review of polarization from the perspective of Jones formalism, where we present two contributions. In the first part, we introduce a new expression of degree of polarization using the spectrum of coherency matrix, precisely first and second order statistics. In the second part, we analyze the intensity of particular case of unpolarized field as a function of polarizer's orientation.
\end{abstract}

Keywords: coherency matrix, electromagnetic, degree of polarization, eigenvalues, polarizer

\section{Introduction}

In wave propagation theory, the dynamics of the wave field can be classified into scalar and vectorial theories. The first case is devoted to the study of the characteristics of the wave field using a scalar representation where the function of space $r$ and time $t$ can be parallel to one of the principal axes of Cartesian coordinates. If the wave field contains more than one component, the study of its dynamics yields to the vectorial representation $[1,2]$. In this latter case, the relation between the different components of the wave gives interesting properties to study and classify, such as the relationship between their amplitudes, their phase variations, their intensities and powers, etc. From this remark, the fundamental metric used to characterize the correlation between the components of the wave field is the polarization [3] which defines the function between the states of different components of the propagating field. For electromagnetic waves, the field variation is perpendicular to the direction of propagation, which means that the electric or magnetic field has two components. Polarization gives information of the different states of the field's components in terms of their amplitudes and phases. Since the properties of the electric field change according to the constraints of the medium of propagation, consequently, the polarization is also changing where, for example, we observe the reduction, the creation and suppression of polarization states.

In this paper, we illustrate some aspects of polarization of the electromagnetic field where we discuss some concepts of polarization [4] related to single monochromatic waves. In the first part, we briefly present the different types of polarization. Next, we introduce a mathematical expression of the degree of polarization using the coherency matrix. This expression is based on the first and second order statistics of eigenvalues. In the last part, we focus on a case study of unpolarized field. We discuss the change of the coherency matrix after the passage of the unpolarized field through a polarizer with different orientations and we compute the corresponding intensity.

Received (November 30, 2017), Review Result (January 6, 2018), Accepted (January 10, 2018) 


\section{Different States of Polarization}

We begin this section by scalar description of a propagating wave. Let us suppose that the variation is parallel to the $x$ axis, and the propagation is in the $z$ direction. Starting from the solution of the Schrodinger equation given by:

$$
\psi=A e^{-i(\vec{p} \cdot \vec{z}-\varepsilon t) / \hbar}
$$

where $A$ is the constant amplitude, $p$ is the momentum of the particle, $\varepsilon$ is the associated energy, and $\hbar$ is the Planck constant, it is possible to transform $\psi$ into the expression of the classical field using the de Broglie relation between momentum and the wave vector $p=\hbar k$ and the relation between the energy and the frequency $v$ as $\varepsilon=2 \pi \hbar v$. We obtain the classical expression of the electric field that verifies Maxwell equations of propagation:

$$
E=E_{x} e^{i(2 \pi v t-\vec{k} \cdot \vec{z})}
$$

The notation of the amplitude is changed from $A$ to $E_{x}$. Let us now suppose that the electric field has two components $E_{x}$ and $E_{y}$ such as:

$$
E=\left(\begin{array}{c}
E_{x} e^{i(2 \pi v t-\vec{k} \cdot \vec{z})} \\
E_{y} e^{i(2 \pi v t-\vec{k} \cdot \dot{z}-\varphi)}
\end{array}\right)
$$

$\varphi$ is the phase difference between the components, $E_{x}$ and $E_{y}$ are the amplitudes in $\mathrm{V} / \mathrm{m}$. Given the variables $\left(E_{x}, E_{y}, \varphi\right)$, several configurations are possible which leads to the definition of the polarization that describes the special cases of the configuration of the variables. The polarization state of the field can be studied by different formalisms such as Stokes parameters [5], Muller matrices [6] and Jones Formalism [7]. Among the tools of polarization classification is the coherency matrix [8]. It is a 2 by 2 Hermitian matrix whose diagonal elements represent the intensities of the field's components. By definition, the coherency matrix is given by the relation:

$$
J=\frac{1}{2} \varepsilon_{0} c<E E^{+}>=\frac{1}{2} \varepsilon_{0} c\left(\begin{array}{cc}
E_{x}^{2} & E_{x} E_{y} e^{i \varphi} \\
E_{x} E_{y} e^{-i \varphi} & E_{y}^{2}
\end{array}\right)
$$

Where $\varepsilon_{0} \square 8.85 \times 10^{-12} \mathrm{~F} / \mathrm{m}$ is the permittivity, $c \cong 3 \times 10^{8} \mathrm{~m} / \mathrm{s}$ is the phase speed, $<f(t)>=(1 / T) \int_{0}^{T} f(t) d t$ is the time average operator and $(.)^{+}$is the conjugate transpose operation, the factor $(1 / 2)$ is included in the equation to have the same result if we use the real notation $\cos (2 \pi v t)$ instead of $e^{i 2 \pi v t}$ because we have $\left.<\cos ^{2}(2 \pi v t)\right\rangle=1 / 2$. $J$ can represent different states of polarization. The first case is linear polarization [8] where the field has only one component or phase difference $\varphi=m \pi$ where $m \in Z$. The horizontal polarization is represented by the matrix:

$$
J_{h}=I_{0}\left(\begin{array}{ll}
1 & 0 \\
0 & 0
\end{array}\right)
$$


$I_{0}=(1 / 2) \varepsilon_{0} c E_{x}^{2}$ is the intensity in $\mathrm{W} / \mathrm{m}^{2}$; similarly the vertical polarization is described by the matrix:

$$
J_{v}=I_{0}\left(\begin{array}{ll}
0 & 0 \\
0 & 1
\end{array}\right)
$$

In the polarization plan, for a field described by the line $E(y)=\tan (\theta) E(x)$ where the components of the field are given by $E(x)=E_{x} \cos (\theta) e^{i 2 \pi v t}$ and $E(y)=E_{x} \sin (\theta) e^{i 2 \pi v t}$ making an angle $\theta$ with the $x$ axis, the coherency matrix is given by:

$$
J_{\theta}=I_{0}\left(\begin{array}{cc}
\cos ^{2}(\theta) & \cos (\theta) \sin (\theta) \\
\cos (\theta) \sin (\theta) & \sin ^{2}(\theta)
\end{array}\right)
$$

The polarization state for particular case of $\theta=45^{\circ}$ is given figure.1.

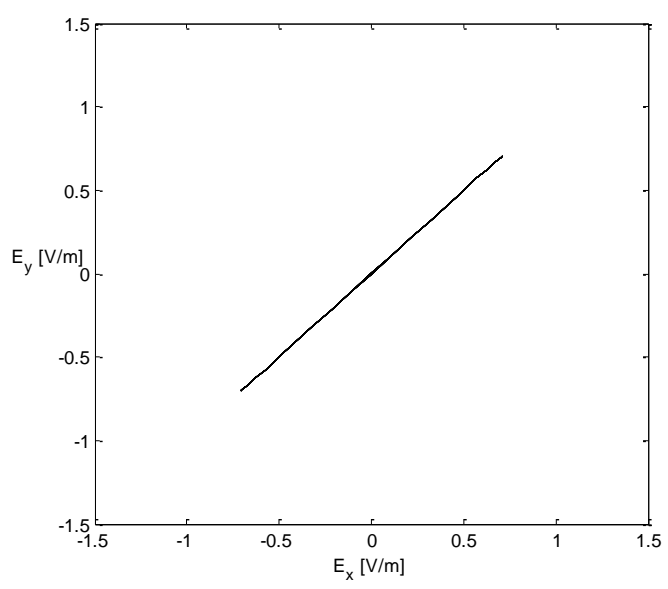

Figure 1. Illustration of Diagonal Polarization

For the particular case of linear polarization oriented by $\theta=45^{\circ}$ and $I_{0}=1 \mathrm{~W} / \mathrm{m}^{2}$, the coherency matrix becomes doubly stochastic which means that all the entries are positive or equal to zero and the sum of any line or column equals unity as $J_{11}+J_{12}=J_{21}+J_{22}=J_{11}+J_{21}=J_{12}+J_{22}=1$. In the case where $E_{x}=E_{y}$ and $\varphi=+\pi / 2$, the field is described by left hand circular polarization [8] and its corresponding matrix is:

$$
J_{\text {lhcp }}=I_{0}\left(\begin{array}{cc}
1 & -i \\
i & 1
\end{array}\right)
$$

Similarly the right hand circular polarization state is defined by phase $\varphi=-\pi / 2$ where $J$ is given by the equation:

$$
J_{r h c p}=I_{0}\left(\begin{array}{cc}
1 & i \\
-i & 1
\end{array}\right)
$$

In Figure.2, we present the polarization plan illustrating the case of circular polarization. 


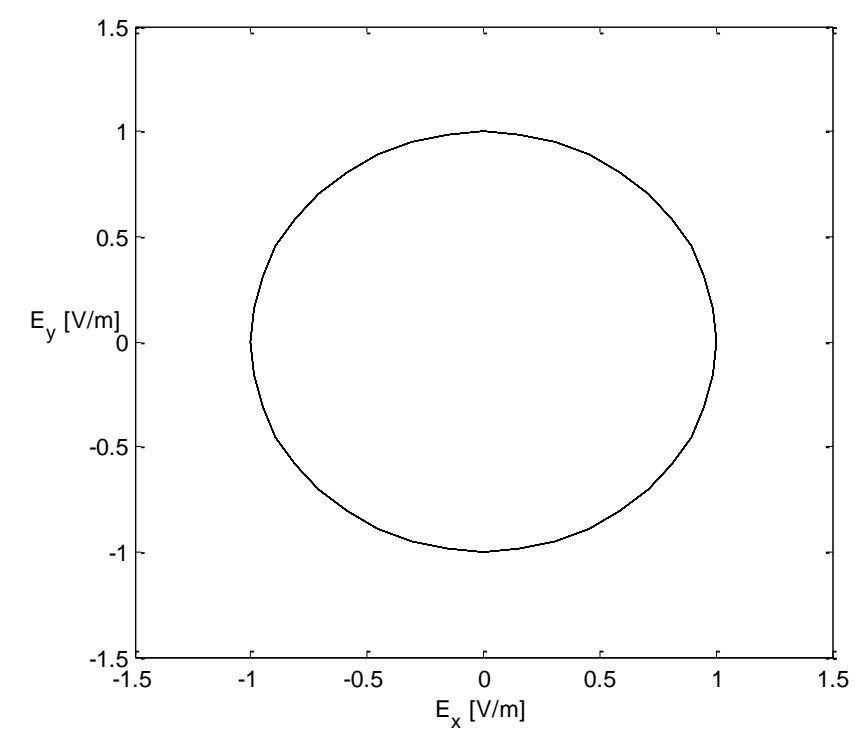

Figure 2. Illustration of Circular Polarization

The elliptical polarization is the generalized case where we have the configuration $E_{x} \neq E_{y}$ and $\varphi \neq 0$ with the condition $\partial \varphi / \partial t=0$. The coherency matrix is therefore:

$$
J_{e l}=\left(\begin{array}{cc}
I_{0 x} & \frac{1}{2} \varepsilon_{0} c E_{x} E_{y} e^{i \varphi} \\
\frac{1}{2} \varepsilon_{0} c E_{x} E_{y} e^{-i \varphi} & I_{0 y}
\end{array}\right)
$$

An illustrative example is given in Figure 3.

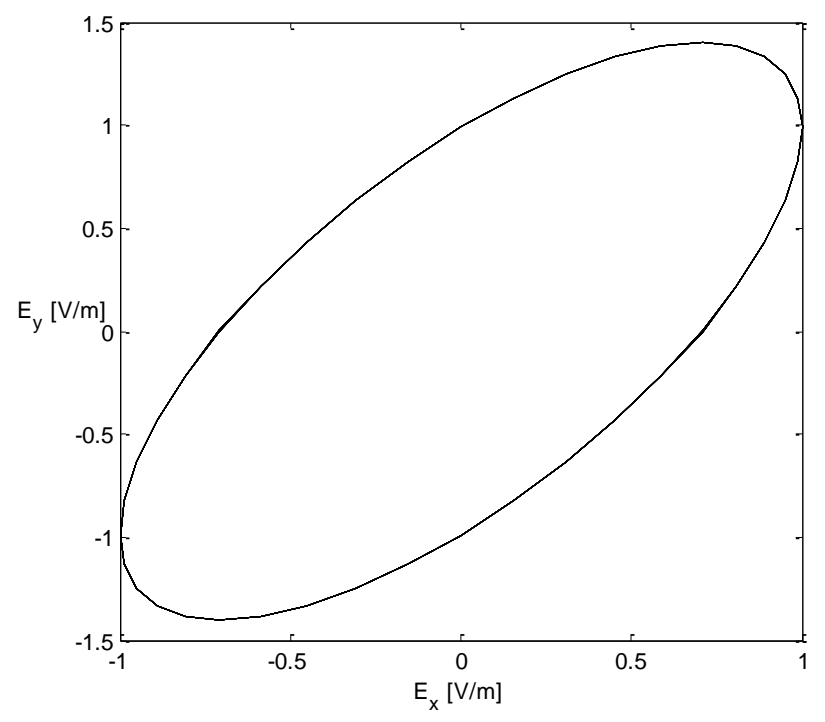

Figure 3. Example of Elliptical Polarization with Parameters

$$
E_{y}=1.4 E_{x} \text { and } \varphi=0.25 \pi
$$


From the above relation we can easily deduce a fundamental property of the coherence matrix, the trace (sum of diagonal elements) is the total intensity of the field [8] $I=\operatorname{Tr}(J)=I_{0 x}+I_{0 y}$. The fourth case is when the angle of polarization is dynamic $\partial \varphi / \partial t \neq 0$. One model of random polarization, as described in [8], is given by the vector:

$$
E=\left(\begin{array}{c}
E_{x}(t) \cos (\varphi(t)) e^{-i 2 \pi v t} \\
E_{x}(t) \sin (\varphi(t)) e^{-i 2 \pi v t}
\end{array}\right)
$$

Where $E_{x}(t)$ varies slowly and $\varphi$ is uniformly distributed in the range $[-\pi,+\pi][8]$. The corresponding coherency matrix is given by:

$$
J_{r p}=\frac{I_{0}}{2}\left(\begin{array}{ll}
1 & 0 \\
0 & 1
\end{array}\right)
$$

It is clear from this model that the components of the field are uncorrelated. An interesting parameter that quantifies the state of polarization can be calculated from the spectrum of $J$ and is called degree of polarization [4] which we discuss in the next section.

\section{Degree of Polarization}

In the previous section, we discussed the four types of polarization states of the electric field which are linear, circular, elliptical and random polarization states. These different states are defined by their corresponding coherency matrix $J$. If we define a parameter defined in the range $[0,1]$ where the value 0 corresponds to the case of a field that is not polarized and 1 for a polarized field such as elliptical, circular or linear, it is possible to extract that parameter based on the spectrum of $J$. Based on the different forms of $J$, a relationship between the eigenvalues can form a single parameter to quantify the polarization state. Since $J$ is a Hermitian operator, its spectral decomposition is possible by the following relation:

$$
J=U \Lambda U^{+}
$$

where $U \in C^{2 \times 2}$ is a unitary matrix such that $U U^{+}=I_{2}$ where $I_{2}$ is the identity matrix, and $\Lambda \in R^{2 \times 2}$ is given by:

$$
\Lambda=\left(\begin{array}{ll}
\lambda_{1} & 0 \\
0 & \lambda_{2}
\end{array}\right)
$$

$\lambda_{1} \geq \lambda_{2}$ are the eigenvalues of $\Lambda$, and the degree of polarization is defined by:

$$
d=\frac{\lambda_{1}-\lambda_{2}}{\lambda_{1}+\lambda_{2}}
$$

The above definition is analogous to the definition of the visibility of light which is the physical interpretation of coherence, where the first eigenvalue represents the maximum intensity and the second eigenvalue represents the minimum intensity. Based on the determinant and trace of matrix $J$, another expression of the degree of polarization is given by [4]: 


$$
d=\sqrt{1-4 \frac{\operatorname{det}(J)}{(\operatorname{Tr}(J))^{2}}}
$$

Where $\operatorname{det}(J)=\lambda_{1} \lambda_{2}$. For the horizontal, vertical and circular polarizations given respectively by $J_{h}, J_{v}$ and $\left(J_{\text {rhcp }}, J_{\text {lhcp }}\right.$ ) we have $d=1$. For elliptical polarization given by $J_{e l}$, the degree of polarization is bounded $0<d<1$, and for non polarized light described by $J_{r p}$ we have $d=0$. For the first contribution, we propose a new expression of the degree of polarization based on the first and second order statistics of eigenvalues. The mean eigenvalue can be written as a function of the trace:

$$
<\lambda>=\frac{\operatorname{Tr}(J)}{2}=\frac{\lambda_{1}+\lambda_{2}}{2}
$$

Next, the variance is written as the following:

$$
\left.(\Delta \lambda)^{2}=<(\lambda-<\lambda>)^{2}>=<\lambda^{2}>-<\lambda\right\rangle^{2}
$$

Given the relation $\left\langle\lambda^{2}\right\rangle=\left(\lambda_{1}^{2}+\lambda_{2}^{2}\right) / 2$, the standard deviation $\Delta \lambda$ can be simplified into the following form:

$$
\Delta \lambda=\frac{\lambda_{1}-\lambda_{2}}{2}
$$

From the equations (17) and (19), the eigenvalues can be expressed using first and second order statistics as the following:

$$
\left\{\begin{array}{l}
\lambda_{1}=<\lambda>+\Delta \lambda \\
\lambda_{2}=<\lambda>-\Delta \lambda
\end{array}\right.
$$

Indeed, the above expression is the special case of the theorem of eigenvalues bounds of symmetric matrices where mean and standard deviation can define the lower and upper bounds of minimal and maximal eigenvalues [9]. If we replace $\lambda_{1}$ and $\lambda_{2}$ by their statistical expressions in the equation of degree of polarization, we obtain the following result:

$$
d=\frac{\lambda_{1}-\lambda_{2}}{\lambda_{1}+\lambda_{2}}=\frac{\Delta \lambda}{<\lambda>}
$$

Therefore, the degree of polarization based on the coherency matrix is the standard deviation over the mean of eigenvalues which is known as the coefficient of variance [10] in information theory. Generally, a high order statistic of eigenvalues, of coherency matrix, if it exists can be calculated using the generalized expression:

$$
\begin{aligned}
<m^{n}> & =<(\lambda-<\lambda>)^{n}>=<\sum_{i=0}^{n} C_{n}^{i} \lambda^{i}(-<\lambda>)^{n-i}> \\
& =\sum_{i=0}^{n} C_{n}^{i}<\lambda^{i}>(-<\lambda>)^{n-i} \\
& =\sum_{i=0}^{n} C_{n}^{i} \frac{\operatorname{Tr}\left(J^{i}\right)}{2}\left(-\frac{\operatorname{Tr}(J)}{2}\right)^{n-i}
\end{aligned}
$$


Given a state of polarization, described by $J$ if the field passes through any instrument that alters the polarization, its coherency matrix changes. Generally, the polarization changes according to the medium of propagation. For example, scattering and diffraction have an impact on the polarization state. As a second contribution in this paper, we investigate in the next section, the polarization state of a particular case of a non polarized field after the passage through a polarizer.

\section{A Case Study of Unpolarized Field}

In this part, we study the change of the coherency matrix of an unpolarized field when it passes through a polarizer. Given an angle $\theta$ relative to the $x$ axis, the corresponding matrix $M$ of the polarizer is given as [8]:

$$
M=\left(\begin{array}{cc}
\cos ^{2}(\theta) & \cos (\theta) \sin (\theta) \\
\cos (\theta) \sin (\theta) & \sin ^{2}(\theta)
\end{array}\right)
$$

The spectrum of $M$ is binary as $\lambda_{1}=1$ and $\lambda_{2}=0$ thus, it is characterized by several properties such as $\operatorname{Tr}(M)=1$ and $M^{n}=M$ for $n \in \square$. The average value $<M>$ as function of rotation angle is diagonal operator, computing the four integrals leads to the following operator:

$$
<M>=\frac{1}{2 \pi} \int_{0}^{2 \pi} M d \theta=\frac{1}{2 \pi}\left(\begin{array}{cc}
\int_{0}^{2 \pi} \cos (\theta)^{2} d \theta & \int_{0}^{2 \pi} \cos (\theta) \sin (\theta) d \theta \\
\int_{0}^{2 \pi} \cos (\theta) \sin (\theta) d \theta & \int_{0}^{2 \pi} \sin (\theta)^{2} d \theta
\end{array}\right)=\frac{1}{2} I_{2}
$$

Given a field described by the Jones vector $E=\left(E_{x}, E_{y}\right)^{T}$, when the field passes through a polarizer, the state of the electric vector changes to $E^{\prime}=M E$, which means that the coherency matrix $J$ is changed to $J$ 'by the following action:

$$
\begin{aligned}
J^{\prime} & =\frac{1}{2} \varepsilon_{0} c<E^{\prime} E^{++}>=\frac{1}{2} \varepsilon_{0} c<M E E^{+} M^{+}> \\
& =\frac{1}{2} \varepsilon_{0} c M<E E^{+}>M^{+}=M J M^{+}
\end{aligned}
$$

We study the effect of the polarizer on a theoretical model of unpolarized light where the field has two components that are uncorrelated and have different magnitudes. The coherency matrix may be represented by:

$$
J=\left(\begin{array}{cc}
I_{x} & 0 \\
0 & I_{y}
\end{array}\right)
$$

Since the components of the field are uncorrelated, the eigenvalues are the diagonal components and the degree of polarization is written in classical form as:

$$
d=\frac{\left|I_{y}-I_{x}\right|}{I_{x}+I_{y}}
$$

Where no polarization implies $I_{x}=I_{y}$, in this part we consider that difference between the intensities is small as $I_{y}=\delta I+I_{x}$, the degree of polarization depends on $\delta I$ and the smaller component $I_{x}$, as the difference tends to zero, the described field tends to be fully non polarized: 


$$
\lim _{\delta I \rightarrow 0} \frac{\delta I}{\delta I+2 I_{x}}=0
$$

By considering a small difference between the components, we study the effect of polarizer on the transmitted intensity using the proposed theoretical model, we choose typical value of degree of polarization that does not exceed 5\%, and the state of polarization is studied using numerical simulation. First we want to derive the expression of the coherency matrix after the passage of the field through the polarizer. Given a polarizer oriented with an angle $\theta$, the coherency matrix of the altered field after the passage is given by:

$$
J^{\prime}=\left(\begin{array}{cc}
\cos ^{4}(\theta) I_{x}+\cos ^{2}(\theta) \sin ^{2}(\theta) I_{y} & \cos ^{3}(\theta) \sin (\theta) I_{x}+\sin ^{3}(\theta) \cos (\theta) I_{y} \\
I_{x} \cos ^{3}(\theta) \sin (\theta)+I_{y} \sin ^{3}(\theta) \cos (\theta) & \cos ^{2}(\theta) \sin ^{2}(\theta) I_{x}+\sin ^{4}(\theta) I_{y}
\end{array}\right)
$$

Therefore, the intensity of the field is:

$$
I=\operatorname{Tr}\left(J^{\prime}\right)=I_{x}\left(\cos ^{2}(\theta) \sin ^{2}(\theta)+\cos ^{4}(\theta)\right)+I_{y}\left(\cos ^{2}(\theta) \sin ^{2}(\theta)+\sin ^{4}(\theta)\right)
$$

The analysis of the state of polarization is based on the variation of the intensity as a function of angle $\theta$ and is performed using theoretical and numerical simulations. In the first part, we conduct a theoretical simulation using the arbitrary values of the intensities $I_{x}=0.8 \mathrm{~mW} / \mathrm{m}^{2}$ and $I_{y}=0.9 \mathrm{~mW} / \mathrm{m}^{2}$, we present in Figure.4, the intensity function $I=f(\theta)$.

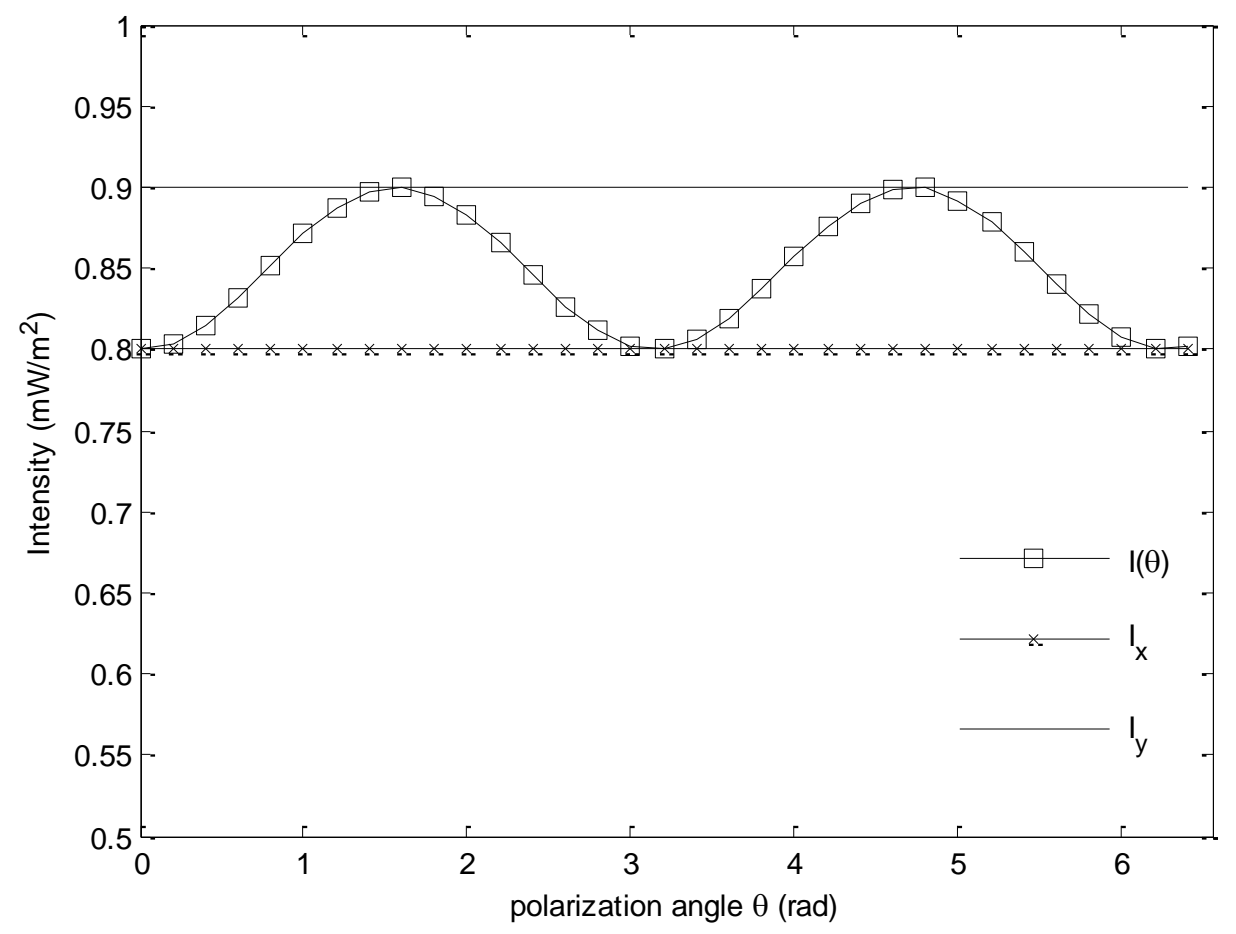

Figure 4. Intensity of Transmitted Unpolarized Field by Polarizer w.r.t Orientation Angle, Theoretical Simulation 
We remark that the variation of the intensity is periodic with average value $(1 / 2)\left(I_{x}+I_{y}\right)$. In the second part, we conduct a numerical simulation using the model proposed by equation (11), for simplicity we consider that the amplitudes of the field are constant. We choose the parameters of the simulation as the following: we consider a carrier wavelength of $\lambda=0.5 \mu \mathrm{m}$ that corresponds to the frequency $v=6 \times 10^{14} \mathrm{~Hz}$. The number of realizations is $K=1000$. We simulate the measurement of the intensity by changing the orientation of the polarizer with step of $d \theta=0.05 \mathrm{rad}$. Each time the polarization angle is changed a new sequence of electric field $E=\left(E_{x}, E_{y}\right)$ is generated where the phase $\varphi$ is randomly changing in the range $[-\pi,+\pi]$. The sequences of the field are generated using the values of the constant amplitudes computed using the relations $E_{x 0}=\sqrt{\frac{4 I_{x}}{\varepsilon_{0} c}}$ and $E_{y 0}=\sqrt{\frac{4 I_{y}}{\varepsilon_{0} c}}$.

In Figure.5, we present the obtained results for the intensity and the estimated degree of polarization.

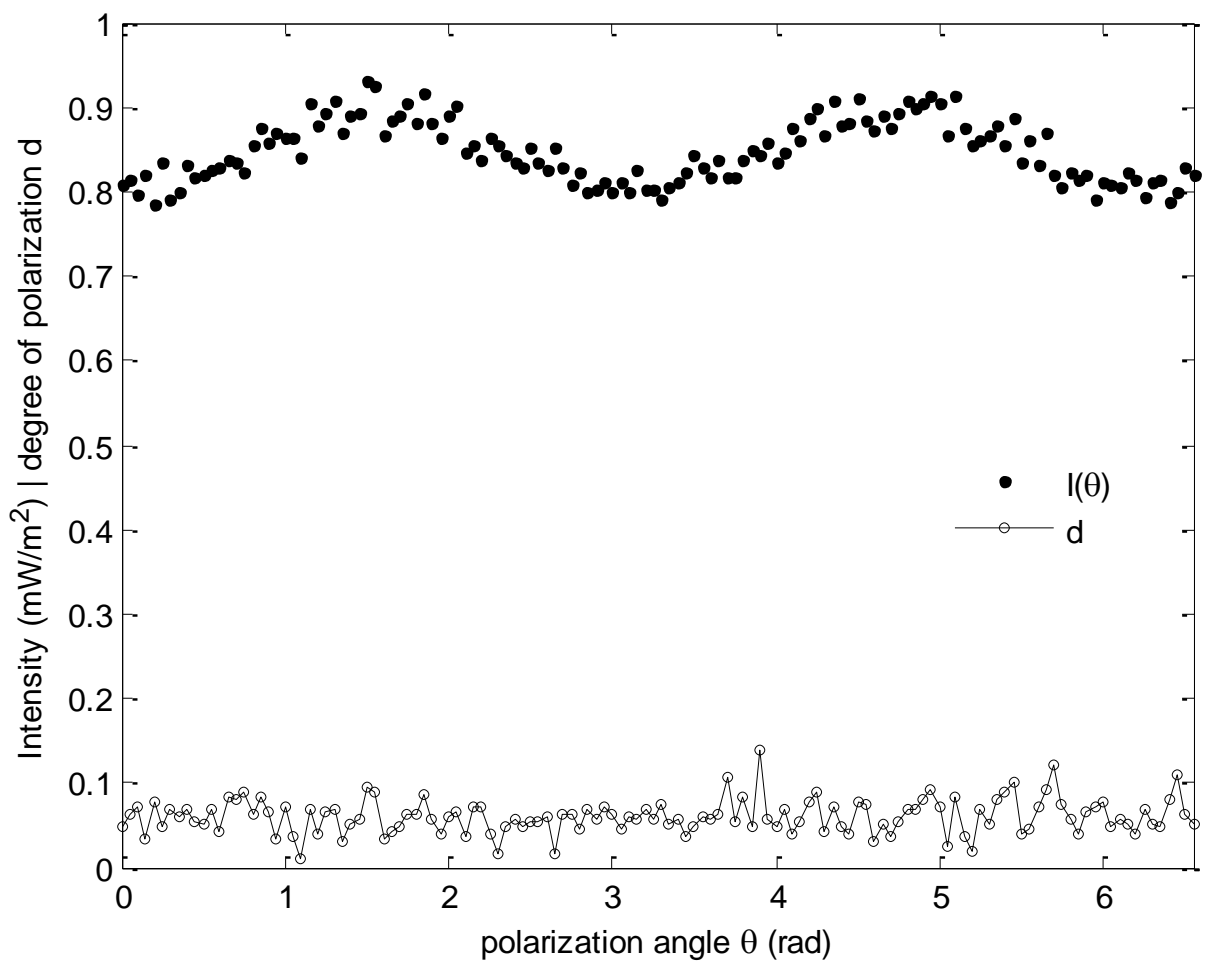

Figure 5. Intensity of Transmitted Unpolarized Field by Polarizer W.R.T Orientation Angle, Numerical Simulation

Note that although $I(\theta)$ and $d$ are given in the same graph, the degree of polarization $d$ is dimensionless parameter. It is obvious that when the polarizer is vertical, the resulting intensity is $I_{y}$ and when the polarizer is horizontal, we only measure the component $I_{x}$, as a last remark, we can verify that if $I_{x}=I_{y}$, we get the usual result [8] of model given by equation (12) where the intensity is independent of the orientation of the polarizer. 


\section{Conclusion}

This paper was devoted to the concept of polarization of electromagnetic field, in the first part, we have presented a brief review of the different states of polarization based on coherency matrix. In the second part, we have introduced a statistical expression of degree of polarization using the eigenvalues of coherency matrix. In the last part, we discussed a particular case of unpolarized field where we have studied the transmitted intensity by polarizer.

\section{References}

[1] H. Guo, J. Chen and S. Zhuang, "Vector plane wave spectrum of an arbitrary polarized electromagnetic wave", Opt. Express, vol. 14, (2006), pp. 2095-2100.

[2] A. Marathay and J. McCalmont, "Vector diffraction theory for electromagnetic waves", J. Opt. Soc. Am., vol. A, no. 18, (2001), pp. 2585-2593.

[3] R. Bhandari, "Polarization of light and topological phases", Physics Reports, ISSN 0370-1573, vol. 281, issue 1, (1997), pp. 1-64.

[4] A. Al-Qasimi, O. Korotkova, D. James and E. Wolf, "Definitions of the degree of polarization of a light beam," Opt. Lett., vol. 32, (2007), pp. 1015-1016.

[5] S. Betti, F. Curti, G. De Marchis and E. Iannone, "Multilevel coherent optical system based on Stokes parameters modulation", in Journal of Lightwave Technology, vol. 8, no. 7, (1990), pp. 1127-1136.

[6] S.-Y. Lu, R. A. Chipman, "Mueller matrices and the degree of polarization", Optics Communications, ISSN 0030-4018, vol. 146, iss. 1-6, (1998), pp. 11-14.

[7] H. Günhan Akarçay, A. Hohmann, A. Kienle, M. Frenz and J. Ricka, "Monte Carlo modeling of polarized light propagation: Stokes vs. Jones. Part I", Appl. Opt., vol. 53, (2014), pp. 7576-7585.

[8] J. W. Goodman, "Statistical Optics", Wiley Classics Library, ISBN 0-47 1-0 1502-4, (2000).

[9] H. Wolkowicz and G.P.H. Styan, "Bounds for eigenvalues using traces, Linear Algebra and its Applications", ISSN 0024-3795, vol. 29, (1980), pp. 471-506.

[10] L. Kostal, P. Lansky and O. Pokora, "Measures of statistical dispersion based on Shannon and Fisher information concepts", In Information Sciences, vol. 235, (2013). 